\title{
TOPOLOGICAL METHODS IN THE THEORY OF FUNCTIONS OF A COMPLEX VARIABLE
}

\author{
MARSTON MORSE AND MAURICE HEINS
}

1. Introduction. Some indication of the reasons for which the authors have undertaken the study of topological methods in the theory of functions of a complex variable is appropriate.

The modern theory of meromorphic functions has distinguished itself by the fruitful use of the instruments of modern analysis and in particular by its use of the theories of integration. Its success along the latter line has perhaps diverted attention from some of the more finitary aspects of the theory which may be regarded as fundamental. In particular, the classical use of the Cauchy integral

$$
\frac{1}{2 \pi i} \int_{C} \frac{f^{\prime \prime}(z)}{f^{\prime}(z)} d z
$$

to find the difference between the number of zeros and poles of $f^{\prime}(z)$ within $C$ is in a sense statistical and ignores important extremal properties of the boundary values such, for example, as the extremal values of $|f(z)|$. In addition, its application requires the existence of $f^{\prime \prime}(z)$, at least almost everywhere on $C$, and the nonvanishing of $f^{\prime}$ on $C$. As we shall see there is a topological substitute for this integral under much weaker conditions on $f$. The needs of the classical theory usually require that the curve $C$ be regular or rectifiable. The topological analogue makes use of Jordan curves whose images under $f$ are "locally simple." See $\$ 3$.

The study of analytic functions is of course paralleled by the study of harmonic functions. An example of a type of mathematical phenomena which the classical theory has failed to reach is given by a harmonic function $U(x, y)$ with infinitely many critical points on a Jordan region $R$. If $U(x, y)$ is continuous on $\bar{R}$, the closure of $R$, there are topological or group theoretic relations between the critical points of $U$ on $R$ and the relative minima of $U$ on the boundary of $R$. These relations take the form of isomorphisms, termed "causal" by the authors, between an appropriately defined group of relative 1-cycles with the $U$-"heights" 1 of the respective "saddle points" of $U$, and a

An address by Marston Morse with discussion by Maurice Heins delivered before the New York meeting of the Society on April 26, 1946, by invitation of the Committee to Select Hour Speakers for Eastern Sectional Meetings; redeived by the editors August 3, 1946.

1 The $U$-height of a cycle is the maximum value of $U(x, y)$ on the cycle. 
group of bounding 0 -cycles with the $U$-heights of the respective relative minima of $U$.

To turn to another aspect of the theory, it is found that a study of harmonic functions on the basis of the topological properties of their level lines leads to a classification of the essential boundary characteristics and to basic relations between these characteristics and the critical points and logarithmic poles of $U$. The logarithmic poles of $U$ are assumed finite in number. This study and the corresponding study of meromorphic functions is of such a general topological nature that the theorems and proofs apply equally well to functions of more general type. The so-called "interior transformations" $f(z)$ generalize the meromorphic functions and "pseudo-harmonic" functions generalize the harmonic functions. These extended functions will presently be defined. The term interior is used essentially in the sense of Stoilow [8]..$^{2}$ In the sense of Whyburn [11], these transformations are "interior" and "light."

In the classical study of meromorphic functions $w=f(z)$ one is usually free to make a conformal transformation of the domain of definitions of $f$, or of the Riemann image. In the study of the zeros, poles, and branch points of interior transformations one can replace conformal transformations by arbitrary homeomorphisms of the domains of both $z$ and $w$. One of the deepest theorems to be reported, Theorem 3.3, was established with the aid of such homeomorphisms after more classical methods failed.

Among the more novel topics which have been studied by the authors is the deformation or homotopy classes of meromorphic functions $f$ with prescribed characteristic sets (zeros, poles, and branch point antecedents). The domain of definition of $f$ has here been restricted to the open disc $|z|<1$, and the number of zeros, poles, and branch point antecedents is fixed and finite. The deformations admitted are through continuous 1-parameter families of meromorphic functions with the same characteristic set. A finite set of numerical invariants $J_{i}(i=1, \cdots, n)$ of $f$ under the admitted deformations has been obtained. The possession of these invariants is a necessary and sufficient condition that two functions $f$ with the same characteristic set be in the same deformation class. In general there is a countably infinite set of deformation classes. In case there is but one zero and no pole, or one pole and no zero in the characteristic set, there is but one deformation class. Up to this point no difference in deformation theory between interior transformations and meromorphic functions exists. In particular, there is a common topological definition of the

2 Numbers in brackets refer to the references cited at the end of the paper. 
invariants $(J)$ which characterize a deformation class. An interior transformation $f$ which possesses the same invariants $(J)$ and the same characteristic set $(\alpha)$ as a meromorphic function $F$ can be admissibly deformed through interior transformations into $F$.

Differences between the theory of interior transformations and meromorphic functions arise, however, if one considers "model" sequences $\left[f_{k}\right]$ of meromorphic functions with a given characteristic set with each $f_{k}$ belonging to a different deformation class. For such a model sequence $\left[f_{k}\right]$ the total set of points $w$ on the $w$-sphere obtained as images under the transformations of $\left[f_{k}\right]$ for $|z|<1$ covers each point of the $w$-sphere infinitely many times, provided the given characteristic set contains both zeros and poles. No such theorem holds for model sequences of interior transformations. The preceding theorem is one of many derivable from the theory of normal families, and the properties of model sequences which we have obtained. It is obviously related to theorems of the Picard type on an essential singularity.

For a more explicit exposition three significant topics have been selected. In terms which presently will be defined these topics are: (1) pseudo-harmonic functions under boundary conditions A, (2) interior transformations with locally simple boundary images, (3) deformation classes of interior or meromorphic functions.

2. Pseudo-harmonic functions under boundary conditions A. Let $u(x, y)$ be a function which is harmonic and not identically constant in a neighborhood $N$ of a point $\left(x_{0}, y_{0}\right)$. Let the points of $N$ be subjected to an arbitrary sense-preserving homeomorphism $T$ leaving $\left(x_{0}, y_{0}\right)$ fixed, carrying $N$ into $N^{\prime}$, and carrying a point $(x, y)$ on $N$ into a point $\left(x^{\prime}, y^{\prime}\right)$ of $N^{\prime}$. Under $T$ set

$$
u(x, y)=U\left(x^{\prime}, y^{\prime}\right) .
$$

The function $U\left(x^{\prime}, y^{\prime}\right)$ will be termed pseudo-harmonic on $N^{\prime}$. The definition will be extended to the case in which $u(x, y)$ has a logarithmic pole at $\left(x_{0}, y_{0}\right)$. In this case $U$, as defined by (2.1), will be said to have a logarithmic pole at $\left(x_{0}, y_{0}\right)$. More generally we admit functions $U(x, y)$ which are pseudo-harmonic, except at most for logarithmic poles, in some neighborhood of each point of a region $G$ (open).We suppose $U$ defined and continuous (except for poles on $G$ ) at every point of $\bar{G}$ (the closure of $G$ ). We shall take $G$ as a finite region bounded by a set of $\nu$ Jordan curves

$$
(B)=\left(B_{1}, \cdots, B_{v}\right) \text {. }
$$

It follows from the known properties of a nonconstant harmonic 
function that the number of level arcs of $U$ which terminate at a point $\left(x_{0}, y_{0}\right)$ of $G$ is even. Set $U\left(x_{0}, y_{0}\right)=c$. A point $(x, y)$ will be said to be above or below $c$ if $U(x, y)>c$ or $U(x, y)<c$ respectively. The level arcs of $U$ terminating at $\left(x_{0}, y_{0}\right)$ divide an appropriately chosen neighborhood of $\left(x_{0}, y_{0}\right)$ into $2 m$ open sectors, alternatingly above and below $c$. If $m=1$, the point $\left(x_{0}, y_{0}\right)$ is ordinary, otherwise critical. When $m>1, m-1$ is called the multiplicity of the critical point $\left(x_{0}, y_{0}\right)$. Critical points of this type will be called saddle points to distinguish them from critical points of $U$ which are relative minima.

The preceding topological characterization of a saddle point of $U$ on $G$ can be extended to points on $(B)$ provided appropriate conditions limit the boundary values of $U$. Under boundary conditions A the function $U^{i}$ defined by $U$ on $B_{i}$ has at most a finite number of points of relative extremum. We assume that $U$ satisfies conditions A.

Let $z_{0}=x_{0}+i y_{0}$ be a point on $B_{i}$. The point $z_{0}$ may be an extremum point of $U$. If this is not the case, it can be shown that there are at most a finite number of level arcs with end points at $z_{0}$, and that these level arcs divide an appropriately chosen neighborhood of $z_{0}$ (relative to $\bar{G}$ ) into a number of sectors, alternatingly above and below $c=U\left(x_{0}, y_{0}\right)$. Let $m$ be the number of these sectors below $c$. If $m=1$, $\left(x_{0}, y_{0}\right)$ is termed ordinary relative to $U$. If $m>1,\left(x_{0}, y_{0}\right)$ is termed a critical or saddle point of $U$ relative to $\bar{G}$, and $m-1$ its multiplicity. Under boundary conditions A the number of saddle points of $U$ on $(B)$ is finite.

A saddle point of $U$ on $(B)$ relative to $\bar{G}$ is not necessarily a saddle point of $-U$. For example, the function $y^{2}-x^{2}$ on the domain $|z-1| \leqq 1$ is ordinary at the point $z=0$, while $x^{2}-y^{2}$ has a saddle point of multiplicity 1 on the same domain.

A basic theorem follows.

TheOREM 2.1. Under boundary conditions A on a pseudo-harmonic function $U$

$$
M-S=2-\nu+s-m
$$

where $M=$ the number of logarithmic poles of $U$ on $G, S=$ the number of saddle points of $U$ on $G, \nu=$ the number of Jordan curves bounding $G$, $s=$ the number of saddle points of $U$ on $(B), m=$ the number of points of relative minimum of $U$ on $(B)$.

Saddle points are counted with their multiplicities.

As might be expected, the proof of this theorem eventually involves the Euler characteristic of the sets $U \leqq c$ and its variation with $c$. However when this formal stage in the proof has been reached the 
major difficulties have all been passed.

In addition to the condition (2.2) one can say that $M+m>0, \nu>0$, $S \geqq 0, s \geqq 0$. These conditions and the condition (2.2) are the only conditions on the integers involved, as one can show by setting up a pseudo-harmonic function realizing any set of integers satisfying these conditions.

If $U(x, y)$ is of class $C^{\prime}$ on a neighborhood of $(B)$, if the boundaries are regular, and $U_{x}$ and $U_{y}$ are never both zero on $(B)$, then $m$ is the number of points of relative minimum of the boundary functions $U^{i}$ at which the gradient vector $g$ of $U$ enters $G$ from $(B)$, and $s$ is the number of points of relative maximum of the functions $U^{i}$ at which $g$ enters $G$ from $(B)$.

Among the cases in which conditions $A$ are not satisfied on a boundary $B_{i}$, the case in which $U$ is constant on $B_{i}$ is important. If $U$ has a constant relative extremum on $B_{i}$, the contribution of $B_{i}$ to the term $s-m$ in (2.2) is null. This happens in the case of a Green's function.

In case $U$ is a constant $c$ on $B_{i}$ but not a relative extremum, the contribution of $B_{i}$ to the term $s-m$ in (2.2) equals one-half the number of arcs at the level $c$ which tend to $B_{i}$ from $G$ on an arbitrarily small neighborhood of $B_{i}$. The proof of the last statement is made on the assumption that $U$ is pseudo-harmonic, except for poles, on a region which includes $\bar{G}$ in its interior, and is continuous on $(B)$.

Theorem 2.1 is the basis of many extensions and applications. For an account the reader is referred to the papers cited. Mention will be made of the fact that one can admit points of singularity $z_{0}$ of $U$ on $G$ in the neighborhood of which

$$
U(x, y)=k \log \left|z-z_{0}\right|+R F(z)
$$

where $k$ is a real constant and $F(z)$ is analytic near $z_{0}$ except for a pole of the $m$ th order at $z_{0}$. In Theorem 2.1 such a point counts as if it were a set of $m+1$ logarithmic poles. One can also suppose that $U$ has a representation of the type (2.3) near a boundary point $z_{0}$ and give a simple rule for the contribution of $z_{0}$ to $s-m$ in (2.2).

3. Interior transformations with locally simple boundary images. A fundamental new instrument here is the locally simple, sensed, closed curve. Such a curve is a topological generalization of the regular curve and in many problems where regular or rectifiable curves are used is more effective and less restrictive.

The curves admitted are continuous and locally 1-1 images $z=z(\theta)$, $0 \leqq \theta \leqq 2 \pi$, of a sensed circle with an angular parameter $\theta$. For such a curve there exists a positive constant $e$ such that any sub-arc of $g$ of diameter less than $e$ is simple; such a constant $e$ is called a norm of 
local simplicity of $g$. A curve can have infinitely many multiple points and still be locally simple. A closed curve which traces a circle $n$ times, $n \neq 0$, in one sense is locally simple.

The angular order $p$ of a regular sensed closed curve $g$ is the number of times a tangent to $g$ rotates through $2 \pi$ as $g$ is traced in its positive sense. If $g$ is merely locally simple, $p$ is defined as follows. Let $k$ be a chord of $g$ which subtends a simple sub-arc of $g$. Let the end points of $k$ trace $g$ in its positive sense remaining distinct and always subtending a simple sub-arc of $g$. The number of times $k$ rotates through $2 \pi$ is independent of the choice of $k$ subject to the above conditions and is termed the angular order $p$ of $g$.

Let $z=a$ be a point not on $g$. The classical order $q(a)$ of $g$ with respect to $a$ is the number of times the vector $z(\theta)-a$ rotates through $2 \pi$ as $g$ is traced in its positive sense.

We shall admit continuous deformations of a locally simple closed curve through a 1-parameter family of such curves, adding the essential condition that the family possess a common norm of local simplicity. The angular order $p$ of a locally simple sensed curve $g$ is invariant under any admissible deformation of $g$. This theorem would not be true if the deformations admitted were through locally simple curves without a common norm of local simplicity. A necessary and sufficient condition that two locally simple sensed curves $g$ be admissibly deformable into each other is that they have the same angular order $p$. In the special case in which the curves are regular this theorem was proved earlier by Whitney and Graustein. See Whitney [10].

A positively sensed circle $C$ traced $n$ times, where $n \neq 0$, will be denoted by $C^{n}$. Let $C^{0}$ denote a figure eight. The angular order of $C^{p}$ is $p$. Thus any locally simple sensed closed curve $g$ can be admissibly deformed into one and only one of the models $C^{p}$. The set of all locally simple sensed closed curves with an angular order $p$ form a deformation class denoted by $c$. It is easy to define the product of two such classes and prove the following theorem:

THEOREM 3.1. The deformation classes $c$ of locally simple sensed closed curves form a group $H$ with respect to multiplication, isomorphic with the additive group $J$ of integers, with a class $c$ in $H$ corresponding to that integer $p$ in $J$ which equals the angular order of the curves in $c$.

The unit class in $H$ contains the figure eight.

Unless otherwise specified, the order $q(0)$ of $g$ will be denoted by $q$. There exist locally simple sensed curves in which $p$ and $q$ are arbitrary integers. An admissible deformation in which no curve intersects the 
origin $z=0$ will be called an $O$-deformation. The pair $(p, q)$ is invariant under any $O$-deformation. Conversely any two locally simple sensed closed curves which have the same invariants $(p, q)$ admit an $O$-deformation into each other. Canonical curves $M_{1}(p, q)$ with the invariants $(p, q)$ are readily defined. $O$-deformation classes and their products can then be defined. The group $H_{1}$ of $O$-deformation classes $c$ with respect to multiplication is isomorphic with the additive group $J_{1}$ of pairs $(p, q)$ of integers, with a class $c$ in $H_{1}$ corresponding to that pair $(p, q)$ in $J$ which yields the orders $(p, q)$ of the curves in $c$. The unit class in $H_{1}$ contains a figure eight neither loop of which encircles the origin.

Interior transformations. Locally simple curves enter naturally into the theory of interior transformations. To define such transformations one begins with a definition of an interior transformation in the neighborhood of an arbitrary point $z_{0}$ of the region $G$ of $\$ 2$. Suppose that $F(t)$ is a nonconstant, meromorphic function defined on a neighborhood $N$ of $t=a$. One subjects $N$ to a 1-1 continuous sense-preserving transformation $t=h(z)$ which maps a neighborhood $N_{1}$ of $a$ onto $N$, holding $a$ fast. The function

$$
F[h(z)]=f(z)
$$

thereby defined on $N_{1}$ is an interior transformation $w=f(z)$ from $N_{1}$ into the complex $w$-sphere. A transformation $w=f(z)$ from $G$ into the $w$-sphere will be called interior if $f$ is an interior transformation of some neighborhood of each point of $G$.

The possibility that $F$ have a pole at $t=a$ is admitted. In that case the function $f$ defined by (3.1) is said to have a pole at $z=a$. We consider interior transformations with at most a finite number of poles on $G$. We suppose that $f$ is defined on $\bar{G}$ and, with respect to the metric of the $w$-sphere, is a continuous transformation from $\bar{G}$ to the $w$-sphere without exception. We do not assume that $f$ is an interior transformation of a neighborhood of points on the boundary $(B)$.

A point $z=a$ of $G$ is termed a branch point antecedent of order $m$ of the inverse $f^{-1}$, if $t=a$ is a branch point antecedent of order $m$ of $F^{-1}$. The order of a zero or pole of $f$ at $z=a$ is similarly defined by reference to $F$.

The basic theorem involves the more novel notion of a partial branch element $E_{m}$ appearing as the Riemann image under $w=f(z)$ of a neighborhood, relative to $\bar{G}$, of a boundary point $z_{0}$. We begin with a model. The continuous Riemann image under the transformation

$$
w-w_{0}=r e^{\theta i}
$$


of the pairs $(r, \theta)$ for which

$$
0 \leqq \theta \leqq(2 m+1) \pi, \quad 0 \leqq r \leqq r_{0} \quad\left(r_{0}>0\right)
$$

will be represented by a Riemann surface spread over a neighborhood of $w_{0}$ in the $w$-plane and termed a partial branch element $E_{m}$ of order $m$. The term is extended to include any Riemann element spread over a neighborhood of $w_{0}$ in the $w$-plane obtained by subjecting $E_{m}$ to a homeomorphism in which $w_{0}$ corresponds to itself and points which cover the same point $w$ retain this property.

The transformation $f$ is termed locally $1-1$ relative to $\bar{G}$ at a boundary point $z_{0}$ if there exists a neighborhood $N$ of $z_{0}$ relative to $\bar{G}$ on which the transformation into the w-plane is a homeomorphism. The principal hypothesis in the following two theorems is that the $w$-images under $f$ of the boundary curves $B_{i}$ are locally simple, ${ }^{3}$ by virtue of the transformation $w=f(z)$. The following theorem gives a condition under which branch point antecedents are isolated on $\bar{G}$.

THEOREM 3.2. If the images (g) under the interior transformation $w=f(z)$ of the boundaries $(B)$ are locally simple, then $f$ is locally 1-1 relative to $\bar{G}$ at all but a finite number of points on $(B)$, and each exceptional point on $(B)$ has a neighborhood relative to $\bar{G}$ whose Riemann image under $f$ is a partial branch element.

In particular if $f$ is meromorphic the theorem implies that the points on $G$ at which $f^{\prime}=0$ are finite in number whenever the boundary images are locally simple.

The main theorem follows:

THEOREM 3.3. If the images ( $g$ ) of the boundaries $(B)$ under the interior transformation $w=f(z)$ are locally simple and do not intersect the point $w=a$, then

$$
2 n(a)=2-\nu+\mu+2 q(a)-p,
$$

where $n(a)=$ the number of zeros of $f(z)-a$ on $G, \nu=$ the number of boundaries $B_{i}, q(a)=$ the sum of the orders of the images $g_{i}$, with respect to $w=a, p=$ the sum of the angular orders of the images $g_{i}, \mu=$ the sum of the orders of the branch elements of $f^{-1}$ including the partial branch elements.

The preceding theorem holds even if $a=\infty$, provided one sets $q(\infty)=0$. One thus has the relation

8 That is, locally simple in terms of the representation acquired under the transformation $w=f(z)$. 


$$
2 n(\infty)=2-\nu+\mu-p .
$$

From (3.3) and (3.4) one obtains the ordinary order relation

$$
n(a)-n(\infty)=q(a) .
$$

A generalization of a theorem of Radó is a particularly simple application of Theorem 3.3. According to Radó there exists no directly conformal $(1, n)$ map $w=f(z)$ of $G$ onto itself when $m>1$ and $\nu>1$. In such a map $n(a)=m$ for each point $a$ of $G$ and $f(z)$ can be continued so as to be continuous on $(B)$ and map each boundary curve $B_{i}$ on some boundary curve $B_{j}$ covered $m$ times. It then follows that $p$ in the theorem is $(2-\nu) m$. Our generalization, which includes Rado's theorem, makes no assumption as to the values of $n(a)$, and the boundary images may intersect themselves and each other. The transformation $f$ is merely interior.

THEOREM 3.4. Under the hypotheses of the preceding theorem with $n(\infty)=0$ and $\nu>2$, it is impossible that $p=(2-\nu) m$ unless $m=1$.

With $p=(2-\nu) m$, and $n(\infty)=0$ it follows from (3.3), on setting $a=\infty$, that $\mu=(\nu-2)(1-m)$. Theorem 3.4 is immediate.

4. Deformation classes of meromorphic functions or interior transformations. We consider sense-preserving interior transformations $w=f(z)$ from the open $\operatorname{disc} S=[|z|<1]$ to the $w$-sphere. We shall restrict ourselves to the case in which $f$ has a finite set of zeros

$$
\left(a_{0}, a_{1}, \cdots, a_{r}\right)
$$

and poles

$$
\left(a_{r+1}, \cdots, a_{n}\right)
$$

with branch point antecedents

$$
\left(b_{1}, \cdots, b_{\mu}\right)
$$

We shall assume that the zeros, poles, and branch points have the order 1, although the case of higher orders can be successfully treated provided the orders do not change during the deformations of $f$. The set

$$
(\alpha)=\left(a_{0}, \cdots, a_{n}, b_{1}, \cdots, b_{\mu}\right)
$$

will be called the characteristic set of $f$.

We shall admit deformations $D$ of $f$ of the form $w=F(z, t)$, defined for $z$ on $S$ and $0 \leqq t \leqq 1$. Here $t$ is the deformation parameter and $F(z, 0) \equiv f(z)$. We require that $F$ map $(z, t)$ continuously into the $w$ - 
sphere and yield an interior transformation of $S$ for each fixed $t$. We shall here report on the case in which the characteristic set is independent of $t$. Deformations of $f$ in which $(\alpha)$ is fixed are termed restricted. In the general theory the sets $(\alpha)$ are permitted to vary over circuits and return to an initial set, or return to the initial set with a permutation of the zeros, poles, and branch point antecedents among themselves. An account of this general theory is included in a paper to be published by the authors [6].

Given an interior transformation $f$ with the characteristic set $(\alpha)$ we shall define a set $(J)$ of $n$ numbers $J_{i}(f, \alpha), i=1, \cdots, n$, associated with the respective pairs $\left(a_{0}, a_{i}\right)$, such that a necessary and sufficient condition that two interior transformations $f_{1}$ and $f_{2}$ defined on $S$ with the same characteristic set $(\alpha)$ be in the same restricted deformation class is that $f_{1}$ and $f_{2}$ have the same set $(J)$.

The difference order $d[k]$. The definition of the invariants $(J)$ involves an extension of the notions of order and angular order in the form of a difference order $d(k)$ defined for a locally simple arc $k$. These arcs are continuous and locally $1-1$ images

$$
w(t)=u(t)+i v(t)
$$

of an interval $\left[0, t_{0}\right]$, and shall intersect fixed end points $w(0)=a$ and $w\left(t_{0}\right)=b$ only when $t=0$ and $t_{0}$ respectively. As previously, a norm $e>0$ of local simplicity of $k$ is a constant such that any sub-arc of $k$ whose diameter is less than $e$ is simple. We shall admit deformations $D$ of $k$ through a continuous family of arcs joining $a$ to $b$ with a common norm of local simplicity; the arcs of the family shall intersect $a$ and $b$ only as end points. Normal forms for such arcs under admissible deformations are desired, together with numerical invariants characterizing such normal forms.

The two cases needed for the definition of the invariants $(J)$ are the cases in which $a=b$ (finite) and the case (referred to the $w$-sphere) where $a$ is finite and $b=\infty$. In case $a=b$ and the arc $w(t)$ is regular, the difference order $d(k)$ is defined as the algebraic increment in

$$
\frac{1}{2 \pi} \arg w^{\prime}(t) \quad\left(0 \leqq t \leqq t_{0}\right)
$$

minus the algebraic increment in

$$
\frac{1}{2 \pi} \arg [w(t)-a] \quad\left(0<t<t_{0}\right)
$$

as $t$ increases from 0 to $t_{0}$, and the argument varies continuously. The limiting directions in (4.4) as $t$ tends to 0 or $t_{0}$ are respectively the 
initial direction in (4.3) and the terminal direction in (4.3) reversed in sense. It follows that $d(k)$ is a half-integer.

In the general case in which $k$ is not regular, $d(k)$ can be readily defined on replacing the tangent vector $w^{\prime}(t)$ in (4.3) by a short chord and appropriately modifying (4.4). In any case $d(k)$ is a half-integer taking on the values

$$
\cdots,-3 / 2,-1 / 2,1 / 2,3 / 2, \cdots \text {. }
$$

A positively sensed circle $C$ through the point $a=b$ has the difference order $1 / 2$. Reversing sense of an arc changes the sign of a difference order. To obtain a model arc $K_{r}$ with a difference order $n=(2 r+1) / 2$ for $r>0$ one attaches a small positively sensed circle $C_{1}$ to $C$ within $C$, with $C_{1}$ tangent to $C$ at some point $a_{1}$ other than $a=b$; one then traces $C$ until $a_{1}$ is reached, then traces $C_{1} r$ times in the positive sense, continuing to $b$ on $C$. Any admissible locally simple arc joining $a$ to itself with a difference order $d(k)$ can be admissibly deformed into the model arc with the same difference order. The difference order is invariant under admissible deformations.

The difference order for the case of a locally simple arc joining $w=a$ to $w=\infty$ is separately defined, but the values of such orders are integers. All integers are realizable as orders as before, and normal forms are readily obtained.

The invariants $J_{i}$. Let $f$ be an interior transformation from $S$ to the $w$-sphere with the characteristic set

$$
(\alpha)=\left(a_{0}, a_{1}, \cdots, a_{n}, b_{1}, \cdots, b_{\mu}\right) \quad(n>1) .
$$

Without loss of generality we can suppose that $a_{0}$ is a zero. (In case there are no zeros we can replace $f$ by its reciprocal.) Let $h_{i}$ be a simple curve joining $a_{0}$ to $a_{i}$ on $S$, and let $h_{i}^{f}$ be the image of $h_{i}$ under $f$. The difference order $d\left(h_{i}^{f}\right)$ will be independent of any continuous deformation of $h_{i}$ through simple arcs joining $a_{0}$ to $a_{i}$ provided $h_{i}$ does not at any time intersect other points of $(\alpha)$. To obtain a number which is independent of the choice of $h_{i}$ among simple arcs joining $a_{0}$ to $a_{i}$ on $S$ we introduce the function

$$
C_{i}(z)=\left[\frac{\left(z-b_{1}\right), \cdots,\left(z-b_{\mu}\right)}{\left(z-a_{1}\right), \cdots,\left(z-a_{n}\right)}\right]\left(z-a_{i}\right) .
$$

Corresponding to a variation of $z$ along $h_{i}$ set

$$
V\left(h_{i}\right)=\frac{1}{2 \pi}\left[\arg C_{i}(z)\right]_{z=a_{0}}^{z=a_{i}} .
$$

The difference 


$$
d\left(h_{i}^{f}\right)-V\left(h_{i}\right)=J_{i}(f, \alpha) \quad(i=1, \cdots, n)
$$

is our definition of $J_{i}$. It is independent of the choice of $h_{i}$ among simple arcs which join $a_{0}$ to $a_{i}$ on $S$ and do not intersect other points of $(\alpha)$, and is invariant under admissible deformations of $f$ since $d\left(h_{i}^{f}\right)$ is so invariant.

It is not in general an integer. But if $F$ is any one admissible interior transformation with the characteristic set $(\alpha)$ and $f$ is any other, then

$$
J_{i}(f, \alpha)=J_{i}(F, \alpha)+r_{i} \quad(i=1, \cdots, n)
$$

where $r_{i}$ is an integer. This follows from the fact that $V\left(h_{i}\right)$ is the same for $f$ and $F$, and that

$$
d\left(h_{i}^{f}\right)-d\left(h_{i}^{F}\right)
$$

is an integer. Each integer $r_{i}$ can be realized by a proper choice of an $f$ with the characteristic set $(\alpha)$. One is thus led to define a countably infinite set of models $f$ corresponding to the countably infinite ensemble of sets $(J)$.

The fundamental theorem here is as follows:

THEOREM 4.1. A necessary and sufficient condition that any two admissible interior transformations $f_{1}$ and $f_{2}$ of $S$ with the same characteristic set belong to the same restricted deformation class is that they possess the same invariants $(J)$. If $f_{1}$ and $f_{2}$ are meromorphic, the deformation can be made through meromorphic functions.

The general proof is in two steps. One first shows that an interior transformation $f$ can be admissibly deformed into a meromorphic function. Then by an interpolatory process the meromorphic function is admissibly deformed into a canonical model $f$ with the given invariants $(J)$. The Stoilow uniformization theorem enters in the first stage of the proof.

The canonical functions $f$. With characteristic set $(\alpha)$ prescribed, the topological theory discloses a countably infinite ensemble of sets $J_{i}, i=1, \cdots, n$, which are invariants of the restricted deformation classes. It remains to establish the existence of a meromorphic function in each deformation class, that is, a meromorphic function with a prescribed characteristic set $(\alpha)$ and a topologically admissible set of invariants $J_{i}$.

To that end one sets

$$
A(z)=\left(z-a_{0}\right)\left(z-a_{1}\right) \cdots\left(z-a_{n}\right), \quad B(z)=\left(z-b_{1}\right) \cdots\left(z-b_{\mu}\right) .
$$


If the required meromorphic function $f$ existed, one could set

$$
\frac{f^{\prime}(z)}{f(z)}=\phi(z) \frac{B(z)}{A(z)}
$$

thereby defining a residual function $\phi(z)$ which, except for removable singularities, is analytic on $S$, never zero, and satisfies the relations

$$
\phi\left(a_{j}\right)=e_{j} \frac{A^{\prime}\left(a_{j}\right)}{B\left(a_{j}\right)} \quad(j=0, \cdots, n)
$$

where $e_{j}=1$ or -1 according as $a_{j}$ is a zero or a pole. One also has the remarkable relation

$$
J_{i}=\left.\arg \phi(z)\right|_{z=a_{0}} ^{z=a_{i}} \quad(i=1, \cdots, n)
$$

where any continuous branch of the argument can be used.

The necessary properties of the residual function $\phi$ as enumerated are characteristic. Corresponding to a prescribed characteristic set $(\alpha)$ and topologically admissible invariants $(J)$ there exists a function $\phi(z)$ which is analytic on $S$, never zero, and satisfies (4.5) and (4.6). Such a function $\phi$ is the residual function of the function

$$
f=K \exp \left\{\int \phi(z) \frac{B(z)}{A(z)} d z\right\} \quad(K \neq 0)
$$

where $K$ is constant. The function $f$ has the prescribed characteristic set and invariants $(J)$.

Covering properties of sequences of meromorphic transformations of $S$. We consider infinite sequences $\left[f_{k}\right]$ of meromorphic transformations of $S$ with the same characteristic set $(\alpha)$ and with no two functions $f_{k}$ in the same restricted deformation class. Such a sequence will be termed a model sequence. We are concerned with the set $W$ of points $w=f_{k}(z), k=1,2, \cdots$, on the $w$-sphere given by a model sequence for $z$ on $S$. When the characteristic set includes both zeros and poles the set $W$ covers each point of the $w$-sphere infinitely many times. When the characteristic set includes no poles, the set $W$ will cover each point of the $w$-sphere infinitely many times ( $w=\infty$ excepted) provided $\left[f_{k}\right]$ does not converge uniformly to zero on every compact subset of $S$.

These results are a consequence of well known theorems on normal families (see Montel [3]) and of the following preliminary lemma. Set $(a)=\left(a_{0}, a_{1}, \cdots, a_{n}\right)$. A model sequence which does not converge 
continuously to 0 or $\infty$ on $S-(a)$ (in the sense of Carathéodory [2, p. 58]) is not a normal family.

The covering theorems stated above for model sequences of meromorphic functions do not hold for sequences of interior transformations. The above covering theorems can be greatly extended and refined in the terms familiar in the theory of normal families. The following theorem is a consequence of Bloch's theorem. See Bieberbach [1, p. 230].

THEOREM 4.2. If $\left[f_{k}\right]$ is a model sequence of meromorphic transformations of $S$, no subsequence of which converges continuously to 0 on $S$, and if the characteristic set $(\alpha)$ includes no poles, there then corresponds to any positive constant $r$, no matter how large, a member $f_{k(r)}$ of the sequence and a circular disc $D_{r}$ of radius $r$ in the w-plane, such that $D_{r}$ is the one-to-one image under $f_{k(r)}$ of some subdomain of $S$.

\section{REFERENCES}

1. L. Bieberbach, Lehrbuch der Funktionentheorie, vol. 2, Leipzig, 1931.

2. C. Carathéodory, Conformal representation, London.

3. Paul Montel, Leçons sur les familles normales de fonctions analytiques, Paris, 1927.

4. M. Morse, The topology of pseudo-harmonic functions, Duke Math. J. vol. 13 (1946) pp. 21-42.

5. M. Morse and M. Heins, Topological methods in the theory of functions of a single complex variable: I. Deformation types of locally simple curves, Ann. of Math. vol. 46 (1945) pp. 600-624; II. Boundary values and integral characteristics of interior transformations and pseudo-harmonic functions, Ibid. pp. 625-666; III. Causal isomorphisms in the theory of pseudo-harmonic functions, Ibid. vol. 47 (1946) pp. 233-274.

6. - Deformation classes of meromorphic functions and their extensions to interior transformations, Acta Math. vol. 80 (1947).

7. T. Rad6, Zur Theorie der mehrdeutigen konformen Abbildungen, Acta Univ. Szeged. vol. 1 (1922) pp. 55-64.

8. S. Stoilow, Leçons sur les principes topologiques de la therorie des fonctions analytiques, Paris, 1938.

9. - Du caractère topologique d'un théorème sur les fonctions méromorphes, C. R. Acad. Sci. Paris vol. 190 (1930) pp. 251-253.

10. H. Whitney, On regular curves in the plane, Compositio Mathematica (1937) pp. 276-284.

11. G. T. Whyburn, Analytic topology, Amer. Math. Soc. Colloquium Publications, vol. 28, New York, 1942.

Institute for Advanced Study and HARVARD UNIVERSITY 